\title{
Cognitive Impairment and Postoperative Outcomes in Patients Undergoing Primary Total Hip Arthroplasty a Systematic Review
}

\section{Citation}

Viramontes, Octavio. 2019. Cognitive Impairment and Postoperative Outcomes in Patients Undergoing Primary Total Hip Arthroplasty a Systematic Review. Doctoral dissertation, Harvard Medical School.

\section{Permanent link}

http://nrs.harvard.edu/urn-3:HUL.InstRepos:41971504

\section{Terms of Use}

This article was downloaded from Harvard University's DASH repository, and is made available under the terms and conditions applicable to Other Posted Material, as set forth at http:// nrs.harvard.edu/urn-3:HUL.InstRepos:dash.current.terms-of-use\#LAA

\section{Share Your Story}

The Harvard community has made this article openly available.

Please share how this access benefits you. Submit a story.

Accessibility 


\title{
Cognitive impairment and postoperative outcomes in patients undergoing primary total hip arthroplasty: A systematic review
}

\author{
Octavio Viramontes (BA) ${ }^{\mathrm{a}}$, Betty M. Luan Erfe (M.D.) ${ }^{\mathrm{b}}$, J. Mark Erfe (M.D. M.P.H.) ${ }^{\mathrm{c}}$, \\ Ethan Y. Brovman (MD) ${ }^{\mathrm{a}, \mathrm{d}, \mathrm{e}}$, Jacqueline Boehme (M.D.) ${ }^{\mathrm{a}, \mathrm{d}}$, Angela M. Bader (M.D., M.P.H) ${ }^{\mathrm{a}, \mathrm{d}}$, \\ Richard D. Urman (M.D. M.B.A) ${ }^{\mathrm{a}, \mathrm{d}, \mathrm{e}, *}$ \\ ${ }^{a}$ Harvard Medical School, Boston, MA, USA \\ ${ }^{\mathrm{b}}$ Internal Medicine Program, Department of Medicine, Queens Hospital Center, New York, NY, USA \\ ${ }^{\mathrm{c}}$ Division of Cardiac Surgery, Department of Surgery, Bluhm Cardiovascular Institute, Northwestern Memorial Hospital, Chicago, IL, USA \\ ${ }^{\mathrm{d}}$ Department of Anesthesiology, Perioperative and Pain Medicine, Brigham and Women's Hospital, Boston, MA, USA \\ ${ }^{\mathrm{e}}$ Center for Perioperative Research, Brigham and Women's Hospital, Boston, MA, USA
}

\section{A R T I C L E I N F O}

\section{Keywords:}

Dementia

Cognitive impairment

Anesthesia

Surgery

Outcomes

Delirium

\begin{abstract}
A B S T R A C T
Study objective: Total hip arthroplasty (THA) is a common surgical procedure in the elderly. Varying degrees of cognitive impairment (CI) are frequently seen in this patient population. To date, there has been no systematic review of the literature specifically examining the impact of CI on outcomes after elective THA. The aim of this systematic review was to identify studies that compare the postoperative outcomes of patients with and without CI after undergoing elective primary THA.

Design: We conducted a systematic review of prospective and retrospective studies. A systematic literature review was conducted by searching MEDLINE, PubMed, and Embase from between January 1, 1997 and January 1, 2018. A total of 234 articles were reviewed and 22 studies were selected.

Setting: Operating room and short-term and long-term postoperative recovery up to 2 years.

Patients: Patients with CI who underwent an elective primary THA that required general anesthesia with a comparator group of patients who did not have dementia.

Interventions: Patients who underwent elective primary total hip arthroplasty.

Measurements: Outcomes included post-operative delirium (POD), mortality and other complications, discharge disposition, length of stay (LOS), mortality, short-term (30 days) and long-term (1 month-2 years) complications. Main results: 22 studies with 5,705,302 participants were included in the systematic review. Sample sizes varied greatly, ranging from 14 to 2,924,995 participants. There was an association between patients with CI and an increase in POD, in-hospital mortality, complications during hospitalization, non-routine disposition, LOS, mortality between 1 month to 2 years, and worse postoperative functional status.

Conclusions: We demonstrate that there are strong associations between patients with pre-existing CI undergoing THA and increased POD, hospital mortality, hospital complications, and hospital LOS. We report good quality evidence linking complications after THA to preexisting CI. Screening for CI can improve care and better predict the risk of developing postoperative complications such as delirium. Further investigations can address perioperative factors that can help reduce complications and show the utility of more widespread assessment of preoperative cognitive impairment.
\end{abstract}

\section{Introduction}

Total hip arthroplasty (THA) is one of the most successful orthopedic procedures designed to alleviate pain and restore function, and is a commonly performed major orthopedic procedure involving the lower extremity [1]. However, patients undergoing THA may suffer major complications, which may be due to surgical, anesthesia or patient-related factors [2]. At the same time, the outcomes of these surgeries have improved in recent decades due to advances in preoperative medical optimization, surgical techniques, anesthetic and postoperative

\footnotetext{
* Corresponding author at: Department of Anesthesiology, Perioperative and Pain Medicine, Brigham and Women's Hospital, Harvard Medical School, Boston, MA 02115, USA.

E-mail address: rurman@bwh.harvard.edu (R.D. Urman).
} 


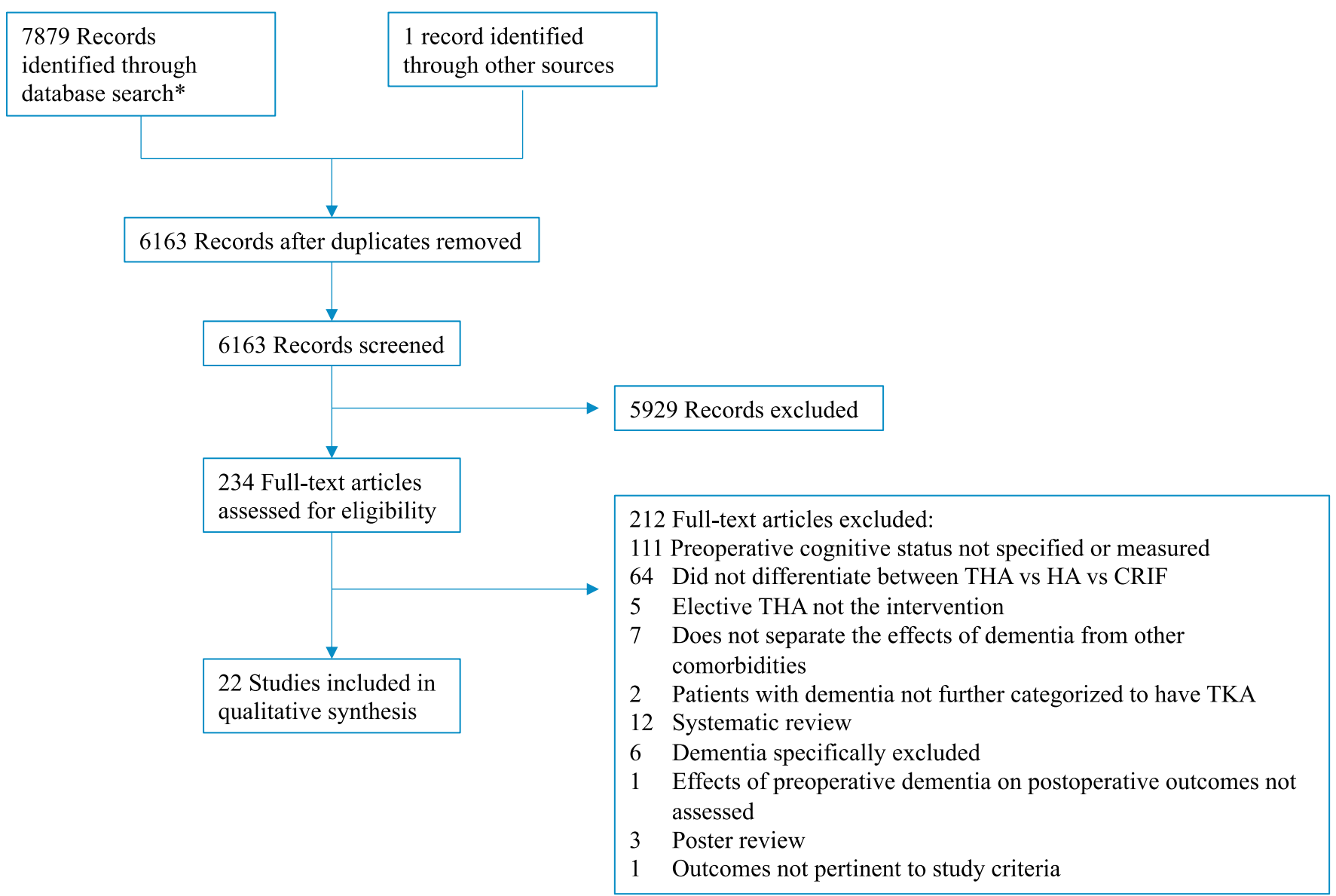

Fig. 1. Flow diagram illustrating the systematic search process to identify relevant studies for inclusion in the final review.

*Articles were identified from PubMed/Medline $(n=4242)$ and Embase $(n=3637)$. See Appendix A for more detail.

THA, total hip arthroplasty; HA, hip arthroscopy; CRIF, closed reduction internal fixation.

care [3]. One way that outcomes have improved has been through risk stratifying patients prior to THA [4]. Previous studies have demonstrated that the presence of cognitive impairment (CI) in patients prior to surgery is associated with poor post-operative outcomes [5]. However, most surgeons and anesthesia providers do not routinely assess cognitive function in THA patients prior to surgery. Given the overall mortality associated with elective THA is estimated at $0.30 \%$ at 30 -days and $0.65 \%$ at 90 -days [6], there exists a need to develop strategies to further decrease major complications in patients undergoing this procedure.

To date, no systematic review has specifically evaluated postoperative outcomes of patients with CI who receive general anesthesia and undergo elective primary THA. A systematic review of prospective, retrospective, and other observational studies can help compare the postoperative outcomes of patients with and without CI after undergoing this procedure. Our hypothesis is that patients with CI will have a more complicated in-hospital course, including higher risk of postoperative delirium, longer hospital stay, and increased wait times to a health care facility. Additionally, we hypothesize that these patients will have an increased risk of mortality and complications within 30 days post-discharge and 30 days to 2 years post-discharge.

\section{Materials and methods}

\subsection{Protocol and registration}

The protocol for this systematic review was designed according to PRISMA guidelines with the assistance of the research librarian at the
Countway Library of Medicine, Boston Massachusetts. The protocol has been registered with PROSPERO (Registration Number: CRD42017072154. Website: http://www.crd.york.ac.uk/PROSPERO).

\subsection{Eligibility criteria}

Original studies that measured post-operative delirium (POD), postoperative mortality, quality of life in patients suffering from preoperatively dementia or cognitive impairment following THA were considered for this review. As there was no single cognitive impairment scale that was used to measure CI, different methods ranging from using Mini-Mental Status Exam (MMSE) in combination with other assessment tools were included in the study. The other tools ranged from National Adult Reading Test (AMNART), Auditory Verbal Learning Test (AVLT), Stroop Color-Word Test (SCWT), and Controlled Word Association Test (COWAT), Mini-Cog( and clock drawing test [7]. However, we only included patients who had CI based on these tools or had a preexisting diagnosis of dementia. Only studies that included patients who underwent elective major total hip arthroplasty were deemed eligible while patients who underwent hemiarthroplasty were excluded. Review articles, published abstracts, letters to the editor, study protocols, and case reports were excluded from this systematic review.

\subsection{Information sources and search}

A literature search was conducted by searching MEDLINE, PubMed, and EMBASE using the following keywords: "Alzheimer's", "dementia", 
"cognition", "cognitive defects", "cognitive deficits", "cognitive disorders", "cognitive dysfunction", "cognitive function", "cognitive impairment", "cognitive status", "memory defects", "memory deficits", "memory disorders", "memory dysfunction", "memory impairment", "mental function", "anesthesia", "surgery", "surgical procedure", "operation", "outcomes", "outcome assessment", "prognosis", and "surgical outcomes". Appendix A provides a detailed description of the database-specific search terms and methodology used during the literature search.

All peer reviewed articles published between January 1, 1997 and January 1, 2018 and published in English were critically evaluated using standards from the PRISMA Statement. Inclusion criteria for this study consisted of (a) patients with stated cognitive impairment who received an elective primary THA that required general anesthesia, and (b) studies focused on adult patients who had a comparator group of patients who did not have dementia. To avoid reporting bias, the only studies included were peer reviewed publications designed as a prospective cohort, retrospective cohort or case-control study. We excluded studies that did not have a comparator group of patients without dementia. Furthermore, we excluded studies that did not evaluate patients for cognitive impairment or specify the patients' cognitive status. If patients had a procedure other than THA or an additional concurrent surgical intervention, those studies were also excluded. Finally, studies that did not observe the outcomes of interest were also excluded. After reviewing all of the searched articles using the parameters mentioned above, a total of 234 studies were reviewed and 22 studies were selected for analysis (Fig. 1). Other reasons for exclusion are listed in Fig. 1. In terms of outcomes measurements, we were interested in inhospital, short- and long-term outcomes. The following outcomes were analyzed for in-hospital outcomes: post-operative delirium (POD), mortality, complications, post-discharge disposition, and length of stay (LOS). The following outcomes were evaluated for short-term outcomes (within 30 days after operation): mortality and complications. For the long-term outcomes (1 month-2 years after an operation) we assessed mortality, functional status, and complications.

\subsection{Study selection and data extraction}

The studies were identified independently and subsequently reviewed by three authors in two different phases. During the first phase, two authors (BLE and JME) conducted, in a systematic way, the review of the articles produced by the search criteria in the databases and appraised study titles and abstracts to determine eligibility. During the second phase, the third author (OV) reviewed the full-text articles identified during the first phase and determined if the articles met the criteria and then included the ones that did. A fourth author (RDU) evaluated the validity of reasons for the excluded articles. In the second phase, the reviewer assessed the study design, duration of follow-up, and the other outcomes of interest. The third author (OV) extracted the data from the studies that met the criteria. The data included publication title, author(s), year of publication, the location of publication, study design, type of patient population, size, intervention given to the patient population, type of cognitive assessment, measure for cognitive impairment, and outcome results.

\subsection{Quality assessment}

The quality and risk of bias of the studies were assessed by the Newcastle-Ottawa-Scale (NOS) [8]. The scale is categorized into three dimensions including selection, comparability, and study type outcome for cohort studies and exposure outcome for case control studies. The scale ranges from 0 to 9 and rates the quality of the studies (ie. good, fair, and poor) by awarding stars in each domain. For example, a "good" quality score required 3 or 4 stars in the selection category, 1 or 2 stars in comparability category, and 2 or 3 stars in outcomes category. A "fair" quality score required 2 stars in selection, 1 or 2 stars in comparability, and 2 or 3 stars in outcomes. A "poor" quality score reflected 0 or 1 star in selection, or 0 stars in comparability, or 0 or 1 star in outcomes. Finally, we used the GRADE (Grading of Recommendations, Assessment, Development and Evaluations) approach to assess the strength of the body of evidence for each study [9]. The analysis of each of the studies based on the Newcastle-Ottawa Scale is shown in Table 2.

\section{Results}

\subsection{Study characteristics}

All of the studies included in this systematic review (Fig. 1) included cohorts of patients who had a preexisting diagnosis of CI or who were found to have CI during pre-operative screening with one of the commonly used screening tools. A total of 22 studies were included. The studies included did not all examine the same postoperative outcomes. Nine of the studies were conducted in the United States, while the other studies included 2 each from Finland and Italy, and 1 study each from the Netherlands, Switzerland, Sweden, France, Taiwan, Turkey, Singapore, Spain, and Germany. All the studies were published during or after 2010. The design of the studies included 7 retrospective cohort studies, 13 prospective cohort studies, and 2 case-control studies. The studies involved a total of 5,705,302 patients undergoing primary elective THA. There were 12 studies undergoing all elective THA, while there were 2 studies of patients undergoing a THA, total knee arthroplasty (TKA) or spine procedure, 7 studies with patients undergoing either a THA or TKA, and 1 study of THA vs other hip surgeries. In terms of the retrospective studies, there were a combined total of $5,701,043$ patients who underwent primary elective THA. Prospective studies had a combined total of 4259 patients who underwent an elective THA.

\subsection{Measures of cognitive impairment}

Cognitive impairment was assessed with multiple scoring systems including the mini-mental status exam (MMSE), National Adult Reading Test (AMNART), Auditory Verbal Learning Test (AVLT), Stroop ColorWord Test (SCWT), and Controlled Word Association Test (COWAT), Mini-Cog $($, clock drawing test, and Cognitive Activity Scale (CAS). Eleven of the 22 studies included patients with a preexisting diagnosis of cognitive impairment. Ten studies utilized the screening tools listed above. There was heterogeneity in the definition of CI, with studies variably using specific cutoff values or assessing cognitive impairment as a continuous linear variable. Studies included in the review are listed in Table 1.

\subsection{Association of cognitive impairment with in-hospital outcomes}

The outcomes examined in the included studies are shown in Table 3. Nine studies assessed the impact of preoperative cognitive impairment on POD. Two of the studies were retrospective cohort studies designed with a combined total of 580 patients who underwent THA. Both studies were good quality studies based on the Newcastle Ottawa scale. The study by Mosk et al. showed that poor preoperative cognitive function predicted POD, while Enemark et al. showed that the rates of POD did not differ from patients who did not have preoperative cognitive dysfunction $[7,8]$.

Seven of the studies were prospective cohort studies with a combined total of 1646 patients who underwent THA. All seven studies were good quality studies based on the Newcastle Ottawa Scale. Zerah et al. showed that POD was more frequent in patients with dementia with a demonstrated odds ratio of $3.12(95 \%$ CI 1.97-4.96, P $=0.05)$ higher than patients without dementia, when controlled for potential confounders [10]. Culley et al. found that $24 \%$ of patients screened positive for probable cognitive impairment using the Mini-Cog@ test score cutoff of less than or equal to 2 [11]. The authors performed an 


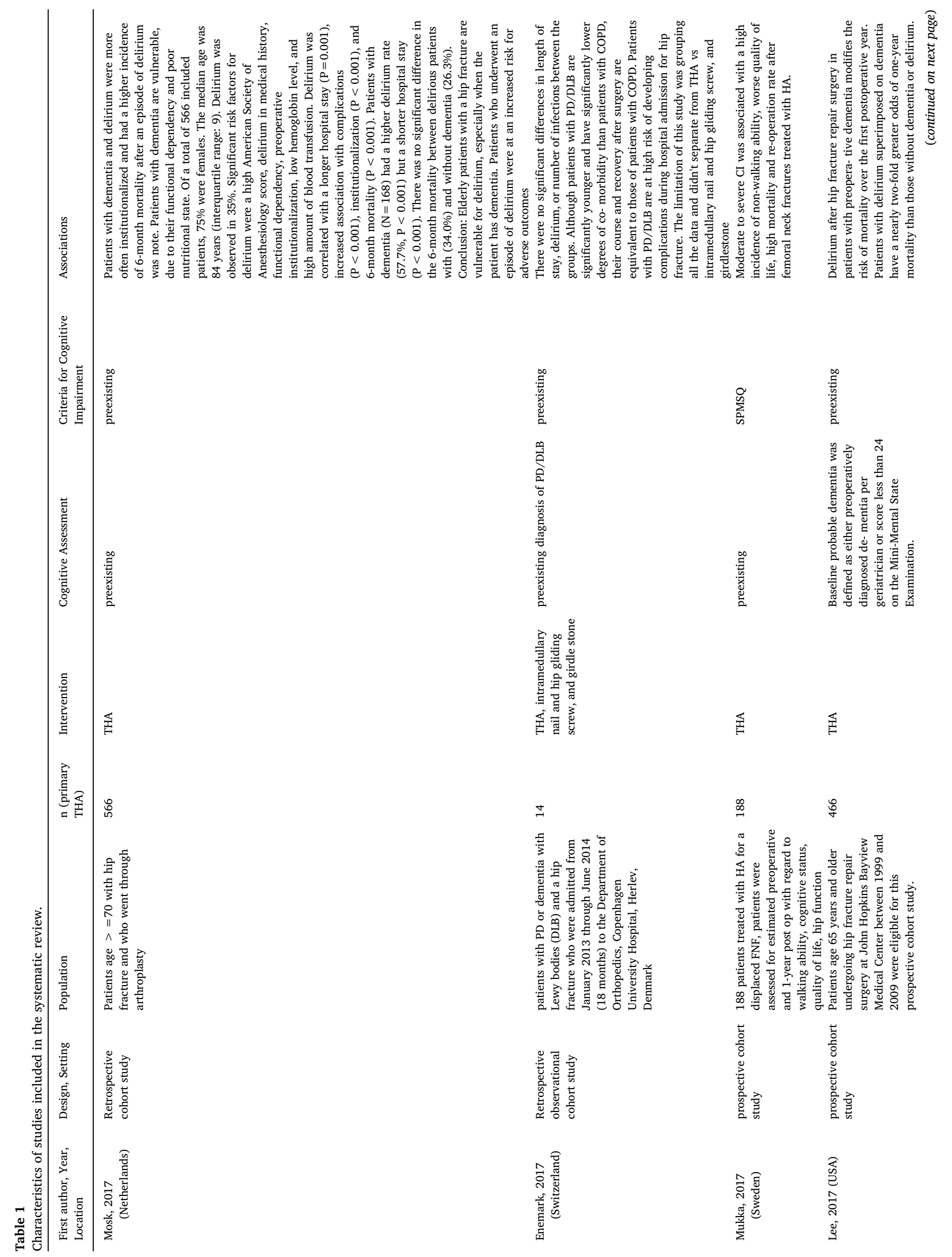




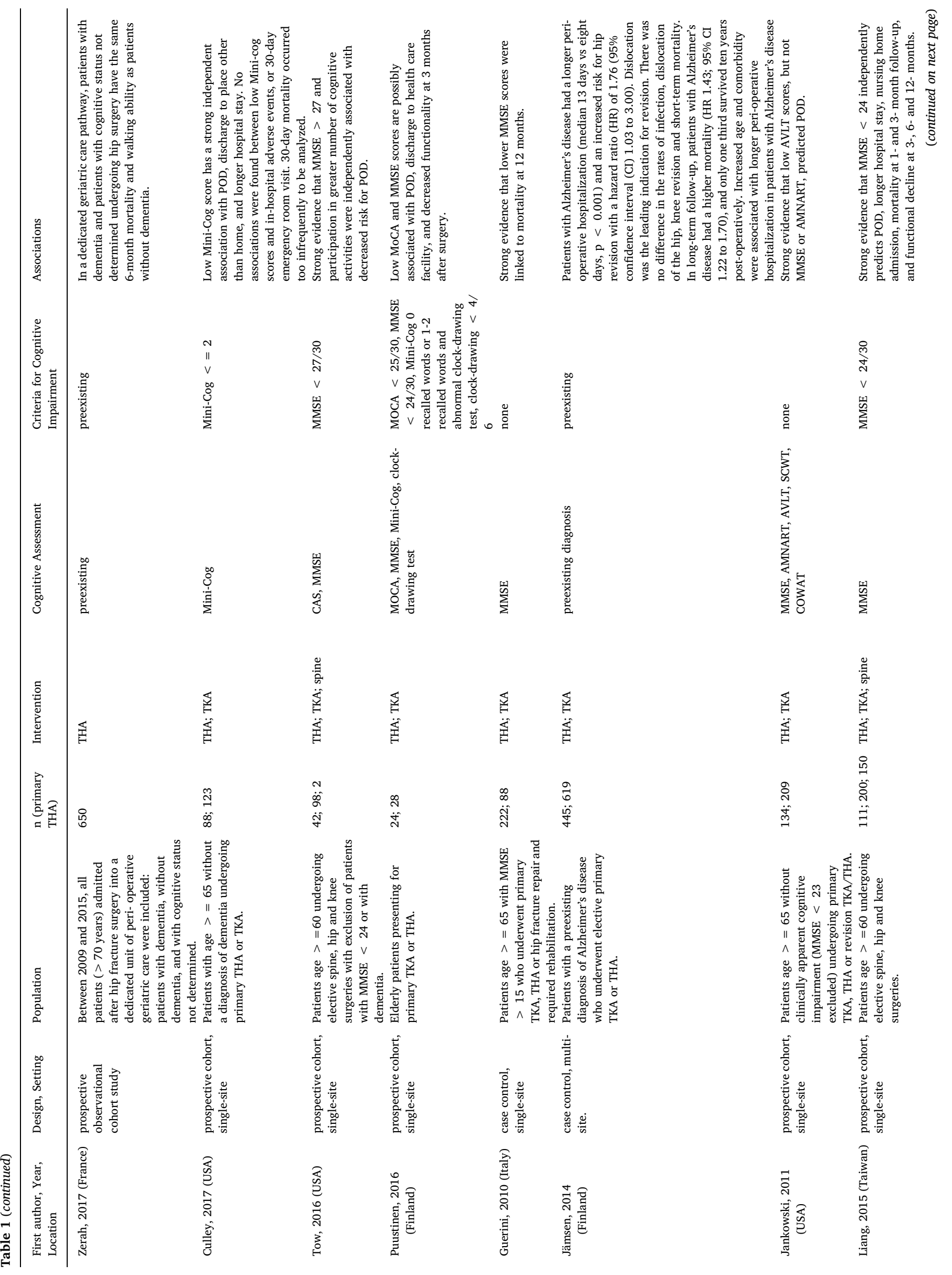




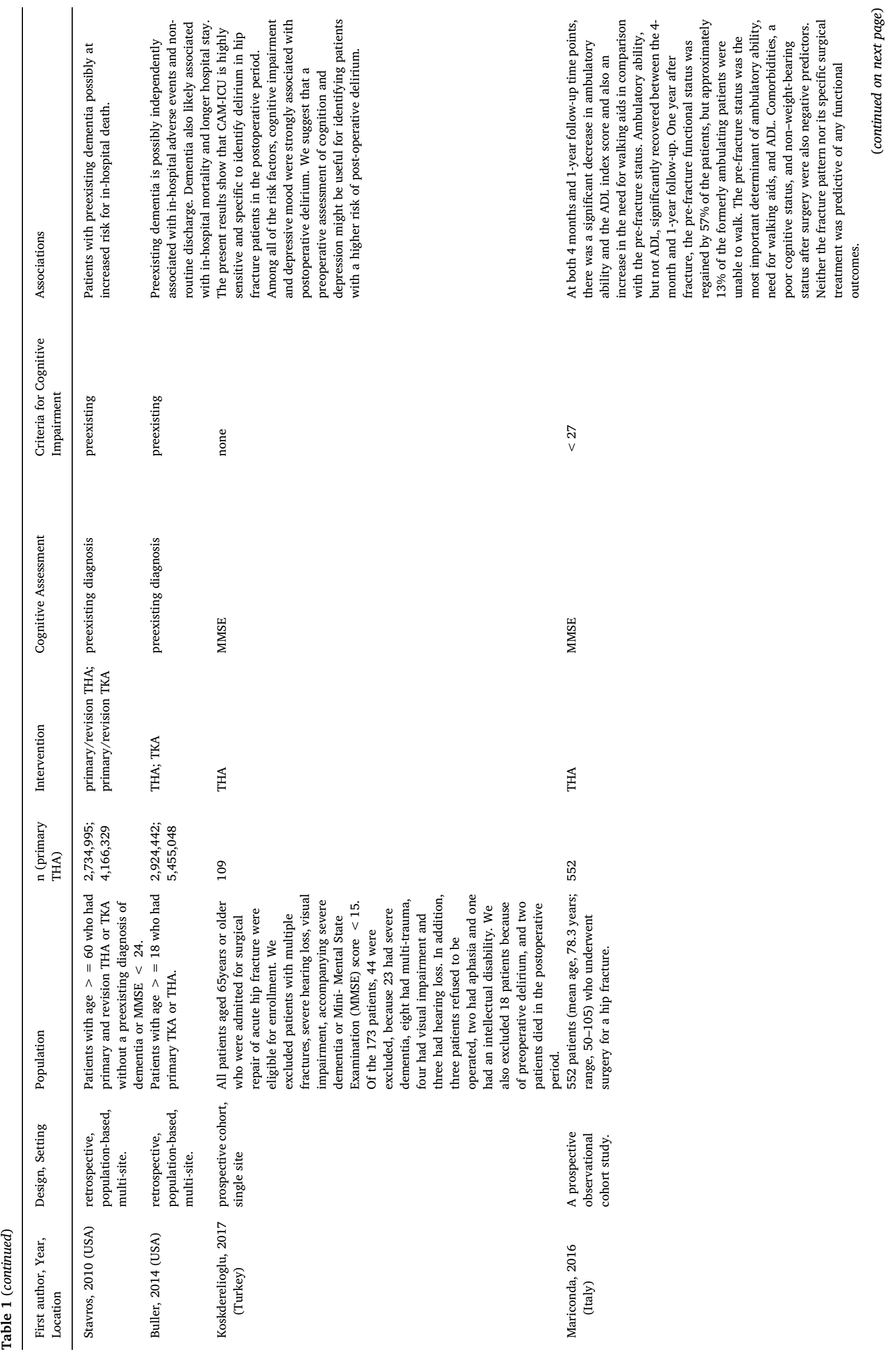




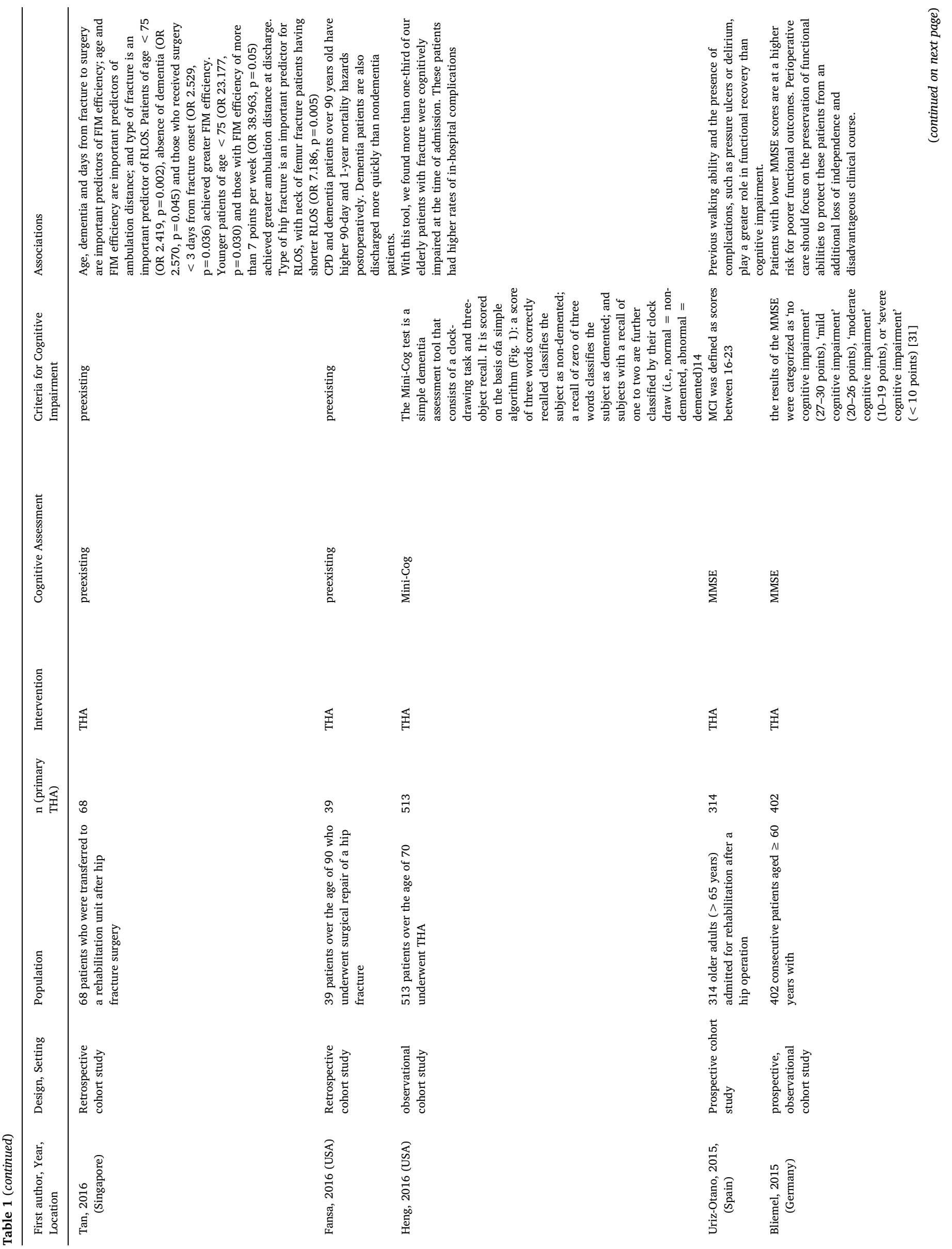




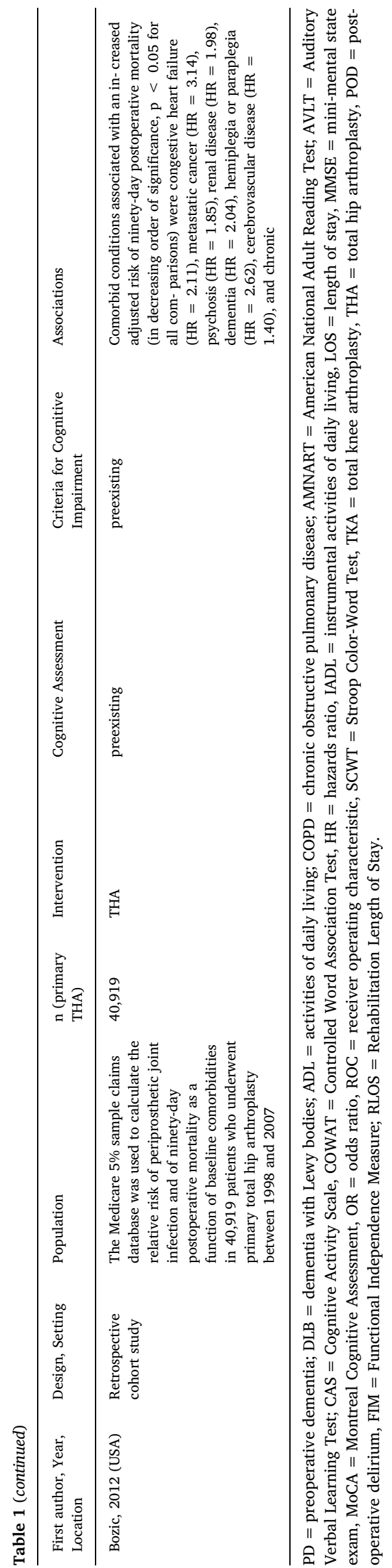

age-adjusted multivariate analysis on patients with a Mini-Cog@ score less than or equal to 2 and showed they were more likely to develop POD with an odds ratio of 4.52 (95\% 1.30-15.68, P = 0.05). Tow et al. measured delirium using Confusion Assessment Method (CAM) and severity Memorial Delirium Assessment Scale (MDAS) to assess for postoperative delirium [12]. The results demonstrated that MMSE scores between 24 and 27 were associated with delirium incidence. Jankowski et al. measured POD using CAM preoperatively and 3 months following surgery [7]. The authors showed that a reduced preoperative neurocognitive status predicted POD. A study by Liang et al. showed that when comparing those who developed post-operative delirium with those who did not, the former had a poorer preexisting cognitive function $(59.5 \%$ vs. $37.0 \%$ of MMSE scores $<24$, $\mathrm{P}<0.007$ ) [13]. Koskderelioglu et al. showed that patients with cognitive impairment defined as a low MMSE score were more likely to have postoperative delirium $(\mathrm{P}=0.001$; odds ratio $0.75,95 \% \mathrm{CI}$ $0.65-0.86$ ) [14]. A study by Heng et al. showed that delirium was significantly increased in patients with an abnormal Mini-Cog® test and had an odds ratio of 3.22 (95\% 1.59-6.77, P = 0.001) [15]. Jamsen et al. was the only case control study with 445 patients who underwent THA that could not confirm an association between preexisting cognitive impairment and POD [16].

Four studies investigated the impact of preoperative cognitive impairment on postoperative in-hospital mortality. Two of the studies were prospective with a combined total of 1052 patients while the remaining were retrospective. All of the prospective studies were good quality studies based on the Newcastle Ottawa Scale. Zerah showed that after adjusting for age, sex, and comorbidities, in-hospital mortality did not differ between dementia patients with a hazard ratio of 0.7 (95\% CI 0.4-1.2), when compared with patients without dementia [10]. Bliemel et al. showed a similar finding, specifically, that complication rates were similar between patients with lower MMSE scores compared to those with normal MMSE scores $(P>0.05)$ [18].

While the retrospective studies had a combined total of 5,659,437 patients. Stavros et al. showed that preoperative risk factors for inhospital mortality were advanced age, presence of comorbid diseases such as dementia, and renal or cerebrovascular disease [17]. The study by Buller et al. showed that patients with a diagnosis of dementia had higher in-hospital mortality $(\mathrm{P}<0.001)$ [19].

Three studies investigated the impact of preoperative cognitive impairment on postoperative in-hospital complications. One of the studies was prospective, while the other 2 were retrospective. The prospective study had a combined total of 513 patients while the retrospective studies had a combined total of $2,924,456$ patients. All studies were good quality studies based on the Newcastle Ottawa Scale. The prospective study of Heng et al. showed that their cohort's rate of in-hospital medical complications was $28.6 \%$ and that patients with cognitive impairment as diagnosed with the Mini-Cog@ test had significantly higher odds of an in-hospital complication compared with patients with a normal Mini-Cog@ (OR, 2.16 [95\% CI 1.42-3.31]; $\mathrm{P}<0.001$ ) [15].

The retrospectives studies such as Buller showed that having diagnoses of depression, dementia and schizophrenia were associated with increased odds of adverse events $(P<0.001)$ [19].

In contrast, Enemark et al. showed that patients with dementia, when compared to the non-dementia group exhibited no significant differences in hospital complications between the two groups [20].

Two studies investigated the impact of preoperative cognitive impairment on discharge disposition. The prospective study had a combined total of 88 patients while the second one was a retrospective study with a combined total of 2,924,442 patients. All these studies were good quality studies based on the Newcastle Ottawa scales. Culley et al. showed that patients with a Mini-Cog@ less than or equal to 2, after adjusting for age and using a multivariate analysis were more likely to be discharged to a place other than home, with an odds ratio of 3.88 (95\% CI 1.58-9.55, P < 0.05) [11]. Buller et al. showed that all 
Table 2

Assessment of study quality using the Newcastle-Ottawa Scale.

\begin{tabular}{lccccc}
\hline First author, year & Selection & Comparability & Outcome & Total & Quality \\
\hline Mosk, 2017 & 4 & 2 & 3 & 9 & Good \\
Enemark, 2017 & 4 & 2 & 3 & 9 & Good \\
Mukka, 2017 & 4 & 2 & 3 & 9 & Good \\
Lee, 2017 & 4 & 2 & 3 & 9 & Good \\
Zerah, 2017 & 4 & 2 & 3 & 9 & Good \\
Culley, 2017 & 3 & 2 & 3 & 8 & Good \\
Tow, 2016 & 4 & 2 & 3 & 9 & Good \\
Puustinen, 2016 & 2 & 0 & 3 & 5 & Poor \\
Guerini, 2010 & 4 & 2 & 3 & 9 & Good \\
Jämsen, 2014 & 4 & 2 & 3 & 9 & Good \\
Jankowski, 2011 & 4 & 2 & 3 & 9 & Good \\
Liang, 2015 & 4 & 2 & 2 & 8 & Good \\
Stavros, 2010 & 4 & 2 & 3 & 9 & Good \\
Buller, 2014 & 4 & 2 & 3 & 9 & Good \\
Koskderelioglu, 2017 & 4 & 2 & 3 & 9 & Good \\
Mariconda, 2016 & 2 & 0 & 3 & 5 & Poor \\
Tan, 2016 & 2 & 0 & 3 & 5 & Poor \\
Fansa, 2016 & 4 & 2 & 3 & 9 & Good \\
Heng, 2016 & 4 & 2 & 3 & 9 & Good \\
Uriz-Otano, 2015 & 4 & 2 & 3 & 9 & Good \\
Bliemel, 2015 & 4 & 2 & 3 & 9 & Good \\
Bozic, 2011 & 4 & 2 & 3 & 9 & Good \\
\hline
\end{tabular}

pre-existing psychiatric comorbidities including dementias were associated with higher odds of non-routine discharge $(P<0.001)$ [19].

Five studies investigated the impact of preoperative cognitive impairment on hospital length of stay. Three studies were retrospective with a combined total of 619 patients, while one was prospective and the last one was a case control study with a combined total of 533 patients. All were good quality studies based on the Newcastle Ottawa Scale. In terms of retrospective studies, the study by Enemark et al. showed that there was no significant difference in length of stay between patients with dementia and patients without the diagnosis [20]. Fansa et al. showed that dementia patients stayed in-hospital postoperatively an average of 5.3 days $(\mathrm{P}=0.013)$ less than non-dementia patients [23]. Mosk et al. showed that patients with dementia has a shorter hospital stay when compared with patients without dementia with a $P$ value $<0.001$ [22]

In contrast, the prospective study by Culley et al. showed that patients with a Mini-Cog( $\bigodot$ less than or equal to 2 were more likely to have longer hospital LOS when compared with patients with scores of $>2$ and showed a hazard ratio of 0.63 (95\% CI 0.42-0.95, P = 0.05) [11]. Jamsen et al. showed that patients with a preexisting diagnosis of Alzheimer's disease had a longer peri-operative hospitalization versus patients who did not have this diagnosis (median 13 days vs eight days, $\mathrm{P}<0.001)$ [21].

\subsection{Association of cognitive impairment with short-term outcomes}

We did not find any good quality studies that investigated the impact of preoperative cognitive impairment on mortality or complications within 30 days.

\subsection{Association of Cognitive Impairment with long-term outcomes}

Six studies investigated the impact of preoperative cognitive impairment on mortality between 1 months and 2 years postoperatively. Four of the studies are prospective studies with a combined total of 1321 patients. Lee et al. showed that patients with dementia had a higher risk of one year mortality than patients without dementia, with a hazard ratio of 1.71 (95\% CI 1.06-2.77, P = 0.05) after adjusting for many variables such as age, sex, medical comorbidity and surgery duration [24]. Guerini et al. showed that patients who were significantly older and had lower cognitive performance died at a higher rate during the 12-month period following discharge. The MMSE mean score for patients who died was 22.3 compared to a mean score of 25.4, which was a significant finding $(P=0.003)$ [25]. Jamsen et al. showed that patients with a preexisting diagnosis of dementia, in that case being Alzheimer's disease, had a higher mortality with a hazard ratio of 1.43 (95\% CI $1.22-1.70, \mathrm{P}=0.05)$ [16]. Mukka et al. showed that patients in the cognitively impaired group had a higher mortality rate when compared with the control group when they used the Kaplan-Meier estimator (log-rank test, $\mathrm{P}=0.0016$ ) [27].

The two retrospective studies included a combined total of 40,958 . Fansa et al. showed that patients with dementia and age over 90 years old have a higher 90 day and 1-year mortality with hazards of $88 \%$ $(P=0.01)$ and $75 \%(P=0.01)$, respectively [23]. Bozic et al. showed that comorbid conditions such as congestive heart failure, metastatic cancer, psychosis, renal disease, hemiplegia or paraplegia, cerebrovascular disease, and chronic pulmonary disease had an increased adjusted risk of 90-day postoperative mortality [26]. Also, dementia had an increased adjusted risk of 90-day postoperative mortality with a hazard ratio of 2.04 (95\% $1.552 .69, \mathrm{P}=0.0001)$.

Four studies investigated the impact of preoperative cognitive impairment on functionality between 1 month and two years postoperatively. Two of the 4 studies were good quality studies while the rest were poor quality based on the Newcastle Ottawa Scale. Three of the four studies are prospective studies with a combined total of 984 patients. Mukka et al. showed that the cognitively impaired group exhibited less function during walking ( $28 \%$ vs. $4 \%$ and with an odds ratio of 9.2 (95\% CI 2.63-32.7, P = 0.001) [27]. Mariconda et al. showed that patients with poor cognitive status with an MMSE score $<27$ had worse prognosis in terms of losing the ability to walk at 1 year and with an odds ratio of 0.93 (95\% CI $0.880 .98, \mathrm{P}=0.005$ ) [29]. Uriz-Otano et al. showed that patients with $\mathrm{CI}$ had lower rates of recovering autonomy in activities of daily living (ADLs) before the surgery and were less likely to walk when compared to patients without cognitive impairment, $50 \%$ vs $73 \%$, respectively $(\mathrm{P}<0.001)$. This study showed that the degree of CI had an odds ratio of 1.12 (95\% CI 1.04-1.22) as it predicted the functional recovery after surgery [30]. While the single retrospective study had a total of 68 patients. Tan et al. showed that patients with dementia had less function post-discharge, and dementia was an important predictor when using the Functional Independent Measure (FIM) tool. Using a univariate analysis they demonstrated that dementia (mean $=1.7 \pm \mathrm{SD} 1.3, \mathrm{P}=0.023$ ) was a factor contributing to a lower FIM score [28].

Three studies investigated the impact of preoperative cognitive impairment on complications between 1 month and two years postoperatively. Two of the studies analyzed were good quality studies while the remaining study by Puustinen et al., was poor quality based on the Newcastle Ottawa Scale [31]. All studies were prospective studies while none were retrospective with a combined total of 1119 patients. Zerah et al. showed that patients with dementia were more likely to be institutionalized after 6 months compared to patients without dementia with an odds ratio of 2.6 (95\% CI 1.4-4.9, $\mathrm{P}=0.003$ ) [10]. Puustinen et al. showed that patients with low preoperative MoCA, MMSE, and Mini-Cog@ scores had higher long-term complications and predicted higher follow-up treatment in health-care center hospitals with an odds ratio of 0.721 with $\mathrm{P}=0.043$ [31]. The Puustinen et al. study was poor quality based on the Newcastle Ottawa Scale. Jamsen et al. showed that patients with a preexisting diagnosis of Alzheimer's disease had an increased risk for hip revision in the long term, with a hazard ratio of 1.76 (95\% CI 1.03-3.00, P = 0.05) [16].

\section{Discussion}

This systematic review demonstrates that there is a fair to good quality evidence demonstrating that preoperative CI predisposes patients undergoing elective primary THA to worse in-hospital outcomes, increasing the risk for delirium, longer hospital stay and discharge to a health care facility rather than home. 
Table 3

Outcome measures assessed in studies meeting inclusion criteria and the strength of the body of evidence for each outcome studied.

\begin{tabular}{|c|c|c|c|c|c|c|c|c|c|c|}
\hline \multirow[t]{2}{*}{ First author, year } & \multicolumn{5}{|l|}{ In-hospital } & \multicolumn{2}{|c|}{ Short-term (within 30 days) } & \multicolumn{3}{|c|}{ Mid-term (1 month-2 years) } \\
\hline & POD & Mortality & Complications & $\begin{array}{l}\text { Discharge } \\
\text { disposition }\end{array}$ & $\begin{array}{l}\text { Length of } \\
\text { stay }\end{array}$ & Mortality & Complications & Mortality & Functionality & Complications \\
\hline Mosk, 2017 & $\mathrm{X}$ & & & & $\mathrm{X}$ & & & & & \\
\hline Enemark, 2017 & $\mathrm{X}$ & & $\mathrm{X}$ & & $\mathrm{X}$ & & & & & \\
\hline Mukka, 2017 & & & & & & & & $\mathrm{X}$ & $\mathrm{X}$ & \\
\hline Lee, 2017 & & & & & & & & $\mathrm{X}$ & & \\
\hline Zerah, 2017 & $\mathrm{X}$ & $\mathrm{X}$ & & & & & & & & $\mathrm{X}$ \\
\hline Culley, 2017 & $\mathrm{X}$ & & & $\mathrm{X}$ & $\mathrm{X}$ & & & & & \\
\hline Tow, 2016 & $\mathrm{X}$ & & & & & & & & & \\
\hline Puustinen, 2016 & & & & & & & & & & $\mathrm{X}$ \\
\hline Guerini, 2010 & & & & & & & & $\mathrm{X}$ & & \\
\hline Jämsen, 2014 & & & & & $\mathrm{X}$ & & & $\mathrm{X}$ & & $\mathrm{X}$ \\
\hline Jankowski, 2011 & $\mathrm{X}$ & & & & & & & & & \\
\hline Liang, 2015 & $\mathrm{X}$ & & & & & & & & & \\
\hline Stavros, 2010 & & $\mathrm{X}$ & & & & & & & & \\
\hline Buller, 2014 & & $\mathrm{X}$ & $\mathrm{X}$ & $\mathrm{X}$ & & & & & & \\
\hline $\begin{array}{l}\text { Koskderelioglu, } \\
2017\end{array}$ & $\mathrm{X}$ & & & & & & & & & \\
\hline Mariconda, 2016 & & & & & & & & & $\mathrm{X}$ & \\
\hline Tan, 2016 & & & & & & & & & $\mathrm{X}$ & \\
\hline Fansa, 2016 & & & & & $\mathrm{X}$ & & & $\mathrm{X}$ & & \\
\hline Heng, 2016 & $\mathrm{X}$ & & $\mathrm{X}$ & & & & & & & \\
\hline Uriz-Otano, 2015 & & & & & & & & & $\mathrm{X}$ & \\
\hline Bliemel, 2015 & & $\mathrm{X}$ & & & & & & & & \\
\hline Bozic, 2011 & & & & & & & & $\mathrm{X}$ & & \\
\hline $\begin{array}{l}\text { Summary of } \\
\text { evidence }^{\mathrm{a}}\end{array}$ & $\begin{array}{l}\text { (4) } 2 \mathrm{~b},(1) \\
4 ; \\
\text { consistent } \\
\text { results }\end{array}$ & $\begin{array}{l}\text { (2) } 2 \mathrm{~b} \text {; } \\
\text { inconsistent } \\
\text { results }\end{array}$ & $\begin{array}{l}\text { (2) } 2 \mathrm{~b} \text {; } \\
\text { inconsistent } \\
\text { results }\end{array}$ & $\begin{array}{l}\text { (3) } 2 b,(1) \\
4 \text {; consistent } \\
\text { results }\end{array}$ & $\begin{array}{l}\text { (3) } 2 \mathrm{~b} \text {, } \\
\text { consistent } \\
\text { results }\end{array}$ & $\begin{array}{l}\text { (2) } 2 \mathrm{~b} \text {; } \\
\text { inconsistent } \\
\text { results }\end{array}$ & $\begin{array}{l}\text { (1) } 2 \mathrm{~b} \text {; } \\
\text { inconsistent } \\
\text { results }\end{array}$ & $\begin{array}{l}\text { (1) } 3 b,(2) \\
2 b ; \\
\text { consistent } \\
\text { results }\end{array}$ & $\begin{array}{l}\text { (2) } 4 \text {, (1) } 2 b \text {; } \\
\text { inconsistent } \\
\text { results }\end{array}$ & (1) $2 b$ \\
\hline $\begin{array}{l}\text { Quality of body } \\
\text { of evidence }^{\mathrm{b}}\end{array}$ & Moderate & Low & Low & Moderate & Moderate & Low & Low & Low & Low & Low \\
\hline
\end{tabular}

a Level of evidence based on Oxford Centre for Evidence-based Medicine's "Levels of Evidence".

b Grading based on the Cincinnati Children's Hospital Medical Center Evidence Collaboration's "Grading the Body of Evidence".

This is the first systematic review to specifically assess preoperative $\mathrm{CI}$ as a prognostic factor for adverse postoperative outcomes in primary THA patients. While previous systematic reviews have examined outcomes in elderly patients admitted with a femoral neck fracture, these studies focused on outcomes after rehabilitation instead of immediately post-operatively [32,33]. In this review we specifically examine patient outcomes immediately after THA in the hospital setting and also include both short- and long-term outcomes rather than just the outcomes following rehabilitation facility stay. Finally, this review included a significantly larger, more diverse cohort of patients than any previous study [32].

Researchers and clinicians are increasingly advocating for the development and use of tools to identify patients with cognitive impairment (CI) who are undergoing surgery. This effort is particularly valuable in the population undergoing THA due to the large number of elderly patients [34]. Screening for CI has been shown to improve care and better predict the risk of developing postoperative complications such as delirium [35]. Post-operative outcomes such as delirium can cause delay in rehabilitation, longer hospitalization stays, increased mortality and possibly long-term cognitive impairment. However, most preoperative evaluations focus on the assessment of cardiac, pulmonary, renal and hepatic organ systems while routine assessment for possible CI is not done [36].

Although previous studies have examined the impact of cognitive impairment and delirium, no existing review has systematically assessed the impact of $\mathrm{CI}$ on outcomes in a large cohort of patients. For example, a review by Bin Abd Razak et al. systematically reviewed the incidence of postoperative delirium in patients undergoing total joint arthroplasty of the hip and knee with the goal of identifying the risk factors that led to higher delirium rates in this patient population [37]. However, there was no analysis of the impact of CI on postoperative outcomes. Similarly, a review by Rao et al. examined the outcomes of patients with dementia versus patients without dementia [38]. However, that study focused on patients with diagnosed dementia, as opposed to cognitive impairment, which is both more prevalent and less clinically apparent.

This review adds to the existing literature by synthesizing the postoperative outcomes based on studies between 1997 and 2018 with patients who had CI and underwent a THA. We were able to assess various post-operative outcomes including POD, mortality, discharge disposition, LOS, as well as short- (e.g. 30 day) and long-term (e.g. 1 month-2 years) complications. For all the outcomes of interest, the majority of the studies show that pre-operative $\mathrm{CI}$ is associated with worse outcomes. However, there is one conflicting finding on the impact of CI on the hospital LOS. Mosk et al. showed that patients with CI had shorter length of stay while the other studies showed the opposite [22]. Potential explanations for this variation include that the patient population of the Mosk et al. study was from a skilled nursing facility and these patients were being discharged back to those facilities.

\subsection{Implications for clinical practice}

The results of our study suggest that $\mathrm{CI}$ is an important and underappreciated factor in patient recovery after elective THA [39]. Routine pre-operative screening currently focuses on organ system dysfunction that may result in immediate complications. Given the impact of CI on postoperative outcomes, patients may benefit from multidisciplinary interventions and clinical pathways to prevent unwanted complications in this population, such as POD. The ready availability of screening instruments and the clinical importance of $\mathrm{CI}$ on outcomes suggests that routine screening for cognitive impairment may be necessary in certain patient populations, such as those aged 65 or older and those with other significant co-existing conditions, in order to risk-stratify these patients [36]. Early identification of patients with CI would allow for focused 
post-operative interventions to limit and mitigate the risk of developing or exacerbating post-operative complications such as delirium or cognitive decline. These patients could be preferentially triaged to perioperative pathways that include early geriatrician involvement, multimodal analgesia, early mobilization and frequent redirection with the goal of maintaining function, limiting delirium and improving neurocognitive recovery.

This study has additional implications for future health services research, as there is evidence that one third of patients with hip fractures have concomitant cognitive impairment, but many hip fracture trials have specifically excluded this population (38). Given the prevalence of $\mathrm{CI}$ and the adverse outcomes associated with the condition, future hip fracture trials are needed to identify factors associated with improved outcomes after elective THA in this higher risk population.

This review also underscores the unavoidable heterogeneity of patients with CI, given variable definitions used in the studies included. Future research is needed to formally subdivide CI, so that trials can be conducted with a more standardized patient cohort. The standardization should be both in measurement methods and the threshold values used to select the CI group to have better comparisons across studies.

\subsection{Strengths and limitations}

This study has several strengths. First, we developed a well-defined protocol with two levels of review, created clear inclusion and exclusion criteria and a good documentation of reasons for study exclusion. We used an established systematic review methodology to evaluate the evidence. Also, we accepted a broad range of terms when it comes to the definition of $\mathrm{CI}$ that ensured the inclusion of all significant published literature. An additional strength is that we also included more recent literature reflecting contemporary surgical and anesthetic practices.

There are several limitations of this study. First, this systematic review is limited since it includes different subgroups of cognitively impaired patients. In addition, this review does not analyze how other covariates such as age or other comorbidities with preoperative cognitive impairment affect the outcomes of this review. Second, there was significant heterogeneity in sample size and study design. For example, the studies employed different tools to assess cognitive status, such as MMSE, Mini-Cog@, MoCA, and several others. Further, the assessment of cognitive status was different in each study as each had different scales and several had a different threshold value. Another limitation to generalizability is the study location. Only nine of the 22 studies were conducted in the US. However, the two largest studies in our review were done in the US and they include $>90 \%$ of the study participants, making this limitation of generalizability less of a concern.

\section{Disclosures}

None.

\section{Acknowledgements}

None.

Appendix A. A detailed description of the database-specific search terms used during the literature search

\section{PUBMED database:}

(alzheimer's OR dementia OR cognition OR "cognitive defects" OR "cognitive deficits" OR

"cognitive disorders" OR "cognitive dysfunction" OR "cognitive function" OR "cognitive

impairment" OR "cognitive status" OR " memory defects" OR "memory deficits" OR "memory

disorders" OR "memory dysfunction" OR "memory impairment" OR "mental function") AND

(anesthesia OR surgery or "surgical procedure" OR operation) AND

(outcomes OR "outcome

assessment" OR prognosis OR "surgical outcomes")

EMBASE and MEDLINE databases:

('alzheimer disease'/exp OR 'alzheimer disease' OR 'dementia'/exp

OR 'dementia' OR 'cognitive

defect'/exp OR 'cognitive defect' OR 'memory disorder'/exp OR 'memory disorder' OR 'mild

cognitive impairment'/exp OR 'mild cognitive impairment' OR

'thinking impairment'/exp OR

'thinking impairment') AND ('anesthesiology'/exp OR 'anesthe-

siology' OR 'surgery'/exp OR

'surgery') AND ('outcomes research'/exp OR 'outcomes research' OR 'outcomes'/exp OR

'outcomes' OR 'outcome assessment'/exp OR 'outcome assessment'

OR 'prognosis'/exp OR

'prognosis') AND ('cognition'/exp OR 'cognition' OR 'mental function'/exp OR 'mental function'

OR 'dementia'/exp OR 'dementia') AND [1997-2017]/py AND [humans]/lim AND [english]/lim

NOT ('children' OR 'child' OR ' pediatric' OR 'adolescent')

\section{References}

[1] Learmonth ID, Young C, Rorabeck C. The operation of the century: total hip replacement. Lancet 2007;370:1508-19.

[2] Young NL, Cheah D, Waddell JP, Wright JG. Patient characteristics that affect the outcome of total hip arthroplasty: a review. Can J Surg 1998;41:188-95.

[3] Opperer M, Danninger T, Stundner O, Memtsoudis SG. Perioperative outcomes and type of anesthesia in hip surgical patients: an evidence based review. World J Orthop 2014;5:336-43.

[4] Courtney PM, Whitaker CM, Gutsche JT, Hume EL, Lee GC. Predictors of the need for critical care after total joint arthroplasty: an update of our institutional risk stratification model. J Arthroplast 2014;29:1350-4.

[5] McIsaac DI, Wijeysundera DN, Huang A, Bryson GL, van Walraven C. Association of the hospital volume of frail surgical patients cared for with outcomes after elective, major noncardiac surgery: a retrospective population-based cohort study. Anesthesiology 2017;126:602-13.

[6] Berstock JR, Beswick AD, Lenguerrand E, Whitehouse MR, Blom AW. Mortality after total hip replacement surgery: a systematic review. Bone Joint Res 2014;3:175-82.

[7] Jankowski CJ, Trenerry MR, Cook DJ, Buenvenida SL, Stevens SR, Schroeder DR, et al. Cognitive and functional predictors and sequelae of postoperative delirium in elderly patients undergoing elective joint arthroplasty. Anesth Analg 2011;112:1186-93.

[8] Wells GA, Shea B, O'Connell D, Peterson J, Welch V, Losos M, et al. The NewcastleOttawa Scale (NOS) for assessing the quality if nonrandomized studies in metaanalyses Available from http://www.ohri.ca/programs/clinical_epidemiology/ oxford.htm, Accessed date: 19 November 2018.

[9] Guyatt GH, Oxman AD, Vist GE, Kunz R, Falck-Ytter Y, Alonso-Coello P, et al. GRADE: an emerging consensus on rating quality of evidence and strength of recommendations. BMJ 2008;336:924-6.

[10] Zerah L, Cohen-Bittan J, Raux M, Meziere A, Tourette C, Neri C, et al. Association between cognitive status before surgery and outcomes in elderly patients with hip fracture in a dedicated orthogeriatric care pathway. J Alzheimers Dis 2017;56:145-56.

[11] Culley DJ, Flaherty D, Fahey MC, Rudolph JL, Javedan H, Huang CC, et al. Poor performance on a preoperative cognitive screening test predicts postoperative complications in older orthopedic surgical patients. Anesthesiology 2017; 127:765-74.

[12] Tow A, Holtzer R, Wang C, Sharan A, Kim SJ, Gladstein A, et al. Cognitive reserve and postoperative delirium in older adults. J Am Geriatr Soc 2016;64:1341-6.

[13] Liang CK, Chu CL, Chou MY, Lin YT, Lu T, Hsu CJ, et al. Developing a prediction model for post-operative delirium and long-term outcomes among older patients receiving elective orthopedic surgery: a prospective cohort study in Taiwan. Rejuvenation Res 2015;18:347-55.

[14] Koskderelioglu A, Onder O, Gucuyener M, Altay T, Kayali C, Gedizlioglu M. Screening for postoperative delirium in patients with acute hip fracture: assessment of predictive factors. Geriatr Gerontol Int 2017;17:919-24.

[15] Heng M, Eagen CE, Javedan H, Kodela J, Weaver MJ, Harris MB. Abnormal mini$\mathrm{cog}$ is associated with higher risk of complications and delirium in geriatric patients with fracture. J Bone Joint Surg Am 2016;98:742-50.

[16] Jamsen E, Peltola M, Puolakka T, Eskelinen A, Lehto MU. Surgical outcomes of hip and knee arthroplasties for primary osteoarthritis in patients with Alzheimer's disease: a nationwide registry-based case-controlled study. Bone Joint J 2015;97B:654-61.

[17] Memtsoudis SG, Della Valle AG, Besculides MC, Esposito M, Koulouvaris P, Salvati EA. Risk factors for perioperative mortality after lower extremity arthroplasty: a 
population-based study of 6,901,324 patient discharges. J Arthroplast 2010;25:19-26.

[18] Bliemel C, Lechler P, Oberkircher L, Colcuc C, Balzer-Geldsetzer M, Dodel R, et al. Effect of preexisting cognitive impairment on in-patient treatment and discharge management among elderly patients with hip fractures. Dement Geriatr Cogn Disord 2015;40:33-43.

[19] Buller LT, Best MJ, Klika AK, Barsoum WK. The influence of psychiatric comorbidity on perioperative outcomes following primary total hip and knee arthroplasty; a 17year analysis of the National Hospital Discharge Survey database. J Arthroplast 2015;30:165-70.

[20] Enemark M, Midttun M, Winge K. Evaluating outcomes for older patients with Parkinson's disease or dementia with Lewy bodies who have been hospitalised for hip fracture surgery: potential impact of drug administration. Drugs Aging 2017;34:387-92.

[21] Jämsen E, Peltola M, Eskelinen A, Puolakka T, Lehto MUK. Hip and knee replacement in patients with neurodegenerative disease: a nationwide register-based casecontrol study. Eur Geriatr Med 2013;4.

[22] Mosk CA, Mus M, Vroemen JP, van der Ploeg T, Vos DI, Elmans LH, et al. Dementia and delirium, the outcomes in elderly hip fracture patients. Clin Interv Aging 2017;12:421-30.

[23] Fansa A, Huff S, Ebraheim N. Prediction of mortality in nonagenarians following the surgical repair of hip fractures. Clin Orthop Surg 2016;8:140-5.

[24] Lee HB, Oldham MA, Sieber FE, Oh ES. Impact of delirium after hip fracture surgery on one-year mortality in patients with or without dementia: a case of effect modification. Am J Geriatr Psychiatry 2017;25:308-15.

[25] Guerini F, Morghen S, Lucchi E, Bellelli G, Trabucchi M. Depressive symptoms and one year mortality among elderly patients discharged from a rehabilitation ward after orthopaedic surgery of the lower limbs. Behav Neurol 2010;23:117-21.

[26] Bozic KJ, Lau E, Kurtz S, Ong K, Rubash H, Vail TP, et al. Patient-related risk factors for periprosthetic joint infection and postoperative mortality following total hip arthroplasty in medicare patients. J Bone Joint Surg Am 2012;94:794-800.

[27] Mukka S, Knutsson B, Krupic F, Sayed-Noor AS. The influence of cognitive status on outcome and walking ability after hemiarthroplasty for femoral neck fracture: a prospective cohort study. Eur J Orthop Surg Traumatol 2017;27:653-8.

[28] Tan YL, Saw HM. Hip fractures: a review of predictors affecting Functional Independence Measure, ambulation and rehabilitation length of stay during inpatient rehabilitation in Singapore General Hospital. Proc Singapore Healthc 2016;25(1):13-8.

[29] Mariconda M, Costa GG, Cerbasi S, Recano P, Orabona G, Gambacorta M, et al. Factors predicting mobility and the change in activities of daily living after hip fracture: a 1-year prospective cohort study. J Orthop Trauma 2016;30:71-7.

[30] Uriz-Otano F, Uriz-Otano JI, Malafarina V. Factors associated with short-term functional recovery in elderly people with a hip fracture. Influence of cognitive impairment. J Am Med Dir Assoc 2015;16:215-20.

[31] Puustinen J, Luostarinen L, Luostarinen M, Pulliainen V, Huhtala H, Soini M, et al. The use of MoCA and other cognitive tests in evaluation of cognitive impairment in elderly patients undergoing arthroplasty. Geriatr Orthop Surg Rehabil 2016;7:183-7.

[32] Muir SW, Yohannes AM. The impact of cognitive impairment on rehabilitation outcomes in elderly patients admitted with a femoral neck fracture: a systematic review. J Geriatr Phys Ther 2009;32:24-32.

[33] Larach MG, Dirksen SJ, Belani KG, Brandom BW, Metz KM, Policastro MA, et al Special article: creation of a guide for the transfer of care of the malignant hyperthermia patient from ambulatory surgery centers to receiving hospital facilities. Anesth Analg 2012;114:94-100.

[34] Smith T, Hameed Y, Cross J, Sahota O, Fox C. Assessment of people with cognitive impairment and hip fracture: a systematic review and meta-analysis. Arch Gerontol Geriatr 2013;57:117-26.

[35] Deo H, West G, Butcher C, Lewis P. The prevalence of cognitive dysfunction after conventional and computer-assisted total knee replacement. Knee 2011;18:117-20.

[36] Sherman JB, Chatterjee A, Urman RD, Culley DJ, Crosby GJ, Cooper Z, et al. Implementation of Routine Cognitive Screening in the Preoperative Assessment Clinic. A A Pract; 2018.

[37] Bin Abd Razak HR, Yung WY. Postoperative delirium in patients undergoing total joint arthroplasty: a systematic review. J Arthroplast 2015;30:1414-7.

[38] Rao A, Suliman A, Vuik S, Aylin P, Darzi A. Outcomes of dementia: systematic review and meta-analysis of hospital administrative database studies. Arch Gerontol Geriatr 2016;66:198-204.

[39] Gabriel RA, Kaye AD, Jones MR, Dutton RP, Urman RD. Practice variations in anesthetic care and its effect on clinical outcomes for primary total hip arthroplasties. J Arthroplast 2016;31:918-22. 